\title{
Resonance Description of S-Nitrosothiols: Insights into Reactivity Supporting Information
}

\author{
Qadir K. Timerghazin, Gilles H. Peslherbe and Ann M. English \\ Centre for Research in Molecular Modeling, and Department of Chemistry and Biochemistry, Concordia \\ University, 7141 Sherbrooke Street West, Montréal, Québec, Canada, H4B 1R6
}

\section{Computational details}

DFT calculations were performed with the hybrid Perdew-Burke-Ernzerhof functional (PBE0), ${ }^{13}$ using an augmented polarization-consistent basis set (aug-pc-1) recently developed by Jensen ${ }^{14}$ and specifically designed for DFT calculations. The aug-cc-pVDZ-PP basis set and the corresponding effective core potential (Peterson, K.A.; Puzzarini C. Theor. Chem. Acc. 2005, 114, 283. Figgen, D.; Rauhut, G.; Dolg, M.; Stoll, H. Chem. Phys. 2005, 311, 227) were used for copper, while the ECP60MWB basis set (with the $g$ polarization function removed) and the corresponding effective core potential (Andrae, D.; Haeussermann, U.; Dolg, M.; Stoll, H.; Preuss, H. Theor. Chim. Acta 1990, 77, 123. Kaupp, M.; Schleyer, P. v. R.; Stoll, H.; Preuss, H.; J. Chem. Phys. 1991, 94, 1360.) were used for iridium.

To determine global minimum energy structures, the geometries of molecules and complexes were optimized with all coordinates relaxed. Subsequent frequency calculations were performed to characterize the nature of the stationary points and obtain harmonic vibrational frequencies. Intermolecular binding enthalpies $\left(\Delta H_{298}\right)$ were calculated with the supermolecule approach, including unscaled zero-point energy and thermal enthalpy $(298 \mathrm{~K})$ corrections. PBE0 calculations were performed with the Gaussian03 package. ${ }^{15}$

NBO and NRT analyses were performed using the NBO 4.M program. ${ }^{16,17}$ For some $\mathrm{CH}_{3} \mathrm{SNO}$ complexes, the NRT procedure produced several resonance structures that provide the same description of the SNO group, but differ in the description of the other bonding interactions. In such cases, the sum of the relative weight of these resonance structures is reported in Table 1.

Full citation for Ref. 15:

Gaussian 03, Revision B.05, Frisch, M. J.; Trucks, G. W.; Schlegel, H. B.; Scuseria, G. E.; Robb, M. A.; Cheeseman, J. R.; Montgomery, Jr., J. A.; Vreven, T.; Kudin, K. N.; Burant, J. C.; Millam, J. M.; Iyengar, S. S.; Tomasi, J.; Barone, V.; Mennucci, B.; Cossi, M.; Scalmani, G.; Rega, N.; Petersson, G. A.; Nakatsuji, H.; Hada, M.; Ehara, M.; Toyota, K.; Fukuda, R.; Hasegawa, J.; Ishida, M.; Nakajima, T.; Honda, Y.; Kitao, O.; Nakai, H.; Klene, M.; Li, X.; Knox, J. E.; Hratchian, H. P.; Cross, J. B.; Bakken, V.; Adamo, C.; Jaramillo, J.; Gomperts, R.; Stratmann, R. E.; Yazyev, O.; Austin, A. J.; Cammi, R.; Pomelli, C.; Ochterski, J. W.; Ayala, P. Y.; Morokuma, K.; Voth, G. A.; Salvador, P.; Dannenberg, J. J.; Zakrzewski, V. G.; Dapprich, S.; Daniels, A. D.; Strain, M. C.; Farkas, O.; Malick, D. K.; Rabuck, A. D.; Raghavachari, K.; Foresman, J. B.; Ortiz, J. V.; Cui, Q.; Baboul, A. G.; Clifford, S.; Cioslowski, J.; Stefanov, B. B.; Liu, G.; Liashenko, A.; Piskorz, P.; Komaromi, I.; Martin, R. L.; Fox, D. J.; Keith, T.; Al-Laham, M. A.; Peng, C. Y.; Nanayakkara, A.; Challacombe, M.; Gill, P. M. W.; Johnson, B.; Chen, W.; Wong, M. W.; Gonzalez, C.; and Pople, J. A.; Gaussian, Inc., Wallingford CT, 2004. 
Structures, Cartesian coordinates (in $\AA$ ) and total energies (in $E_{h}$ ) for the molecules reported in Table 1.

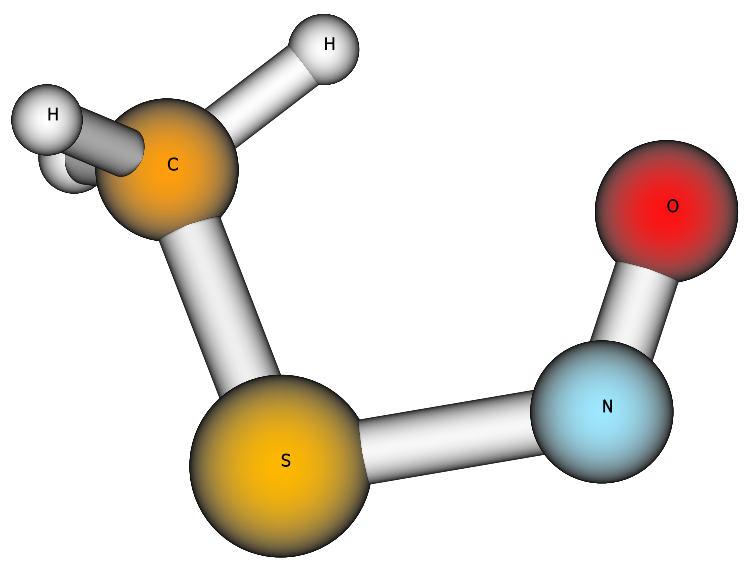
Cis $-\mathrm{CH}_{3}-\mathrm{SNO} \mathrm{E}=-567.6519059$
O, -1.281253 ,
0.000000
$-1.125518$
$\mathrm{N},-1.280176$
0.000000,
0.053888
S, 0.340007 ,
0.000000,
0.882247
C, 1.460951,
0.000000,
$-0.511771$
$\mathrm{H}, 0.834820$,
0.000000 ,
$-1.418421$
$\mathrm{H}, 2.085309$,
-0.898447 ,
$-0.499987$
$\mathrm{H}, 2.085309$,
0.898447 ,
$-0.499987$ 


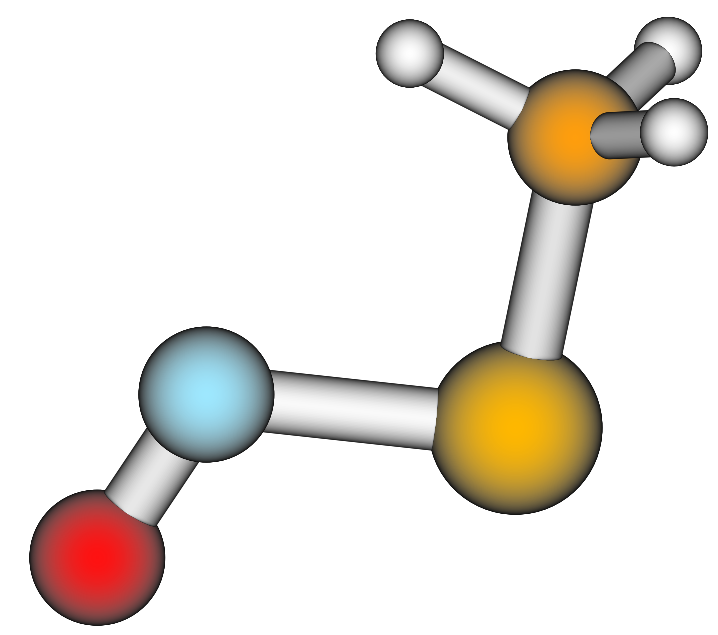

trans $-\mathrm{CH}_{3}-\mathrm{SNO} \mathrm{E}=-567.6501555$

$0,0,-0.9596726052,0 .,-1.7964461266$

$\mathrm{N}, 0,-0.8965960063,0 .,-0.6226391318$

$S, 0,0.7797460679,0 ., 0.0956671451$

$\mathrm{C}, 0,0.2082655197,0 ., 1.7994119809$

$\mathrm{H}, 0,-0.8896200602,0 ., 1.7648587714$

$\mathrm{H}, 0,0.558821371,0.8964742139,2.3190189788$

$\mathrm{H}, 0,0.558821371,-0.8964742139,2.3190189788$ 


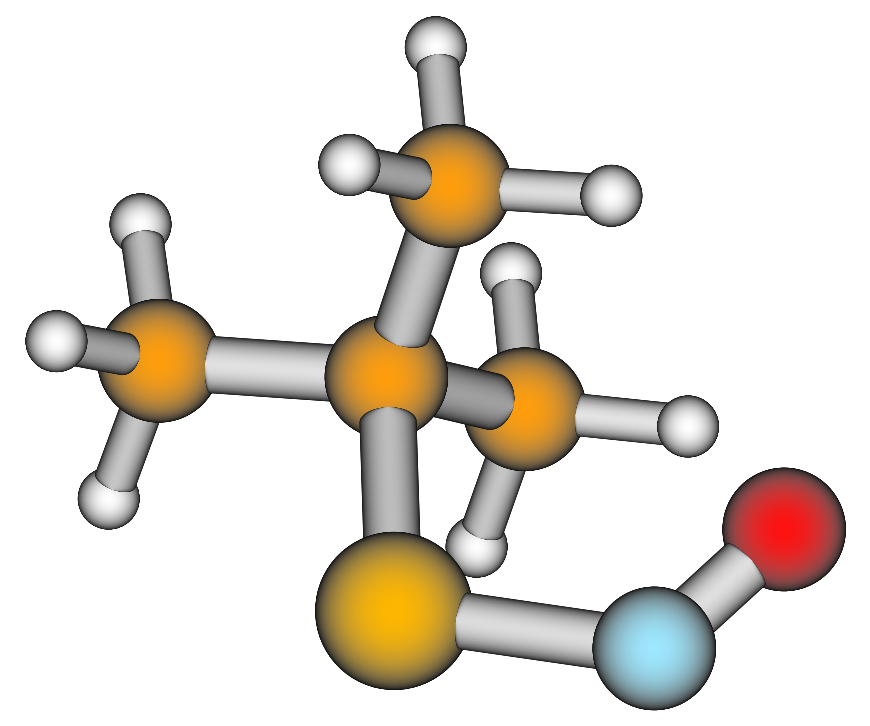

Cis- $\left(\mathrm{CH}_{3}\right)_{3} \mathrm{C}-\mathrm{SNO} \mathrm{E}=-685.4306096$

$0,0,-2.1921247661,0 .,-0.5912030854$

$\mathrm{N}, 0,-2.0098997751,0 ., 0.5820420192$

$\mathrm{S}, 0,-0.3325999175,0 ., 1.1928860926$

$\mathrm{C}, 0,0.8256131644,0 .,-0.2550595209$

$\mathrm{C}, 0,2.2143466628,0 ., 0.3832118603$

$\mathrm{C}, 0,0.6219796669,-1.2649598132,-1.0829515183$

$\mathrm{C}, 0,0.6219796669,1.2649598132,-1.0829515183$

$\mathrm{H}, 0,-0.3773817764,1.3045826099,-1.5251855928$

$\mathrm{H}, 0,1.3560574495,1.2790740608,-1.9007408492$

$\mathrm{H}, 0,0.7743361714,2.1624161986,-0.4732954381$

$\mathrm{H}, 0,-0.3773817764,-1.3045826099,-1.5251855928$

$\mathrm{H}, 0,1.3560574495,-1.2790740608,-1.9007408492$

$\mathrm{H}, 0,0.7743361714,-2.1624161986,-0.4732954381$

$\mathrm{H}, 0,2.9711744659,0 .,-0.4129297981$

$\mathrm{H}, 0,2.3735910566,0.8901571067,1.0035154038$

$\mathrm{H}, 0,2.3735910566,-0.8901571067,1.0035154038$ 


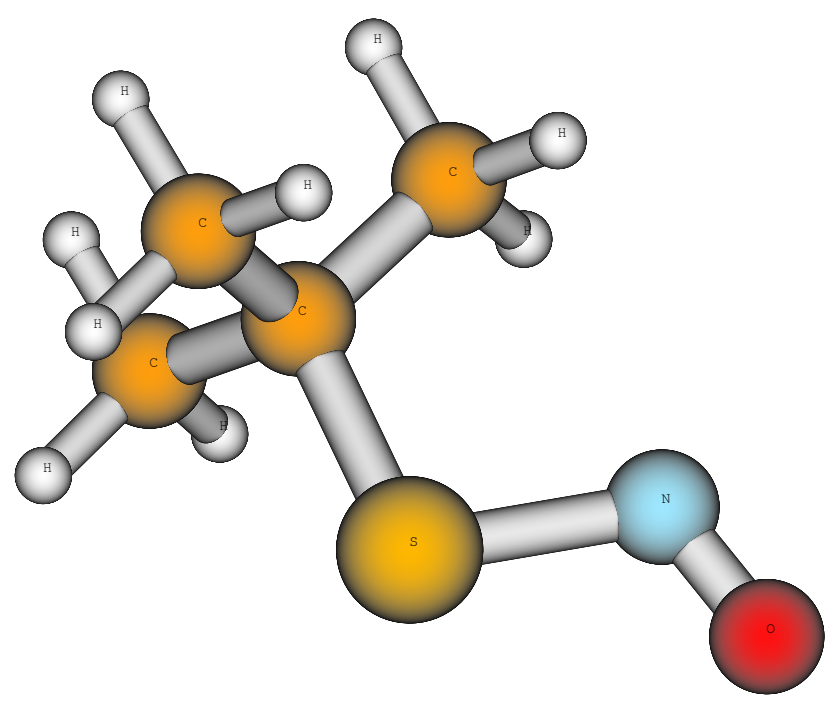

trans $-\left(\mathrm{CH}_{3}\right)_{3} \mathrm{C}-\mathrm{SNO} \mathrm{E}=-685.429605$

$0,-2.3633827133,0 ., 1.7444467506$

$\mathrm{N},-1.2308481144,0 ., 1.4069850419$

$S,-0.9750283885,0 .,-0.3835712428$

$\mathrm{C}, 0.8795729099,0 .,-0.4842122936$

$\mathrm{C}, 1.5205163853,0 ., 0.8961938366$

$C, 1.2757359639,1.2550350509,-1.2594289325$

C, $1.2757359639,-1.2550350509,-1.2594289325$

$\mathrm{H}, 0.793556624,-1.2875698659,-2.243845864$

$\mathrm{H}, 0.9983138779,-2.1622043962,-0.7117177435$

$\mathrm{H}, 2.3633477621,-1.2614840715,-1.4156242215$

$\mathrm{H}, 0.793556624,1.2875698659,-2.243845864$

$\mathrm{H}, 0.9983138779,2.1622043962,-0.7117177435$

$\mathrm{H}, 2.3633477621,1.2614840715,-1.4156242215$

$\mathrm{H}, 2.6103021826,0 ., 0.7610294155$

$\mathrm{H}, 1.246673337,0.8858072687,1.4776373812$

$\mathrm{H}, 1.246673337,-0.8858072687,1.4776373812$ 


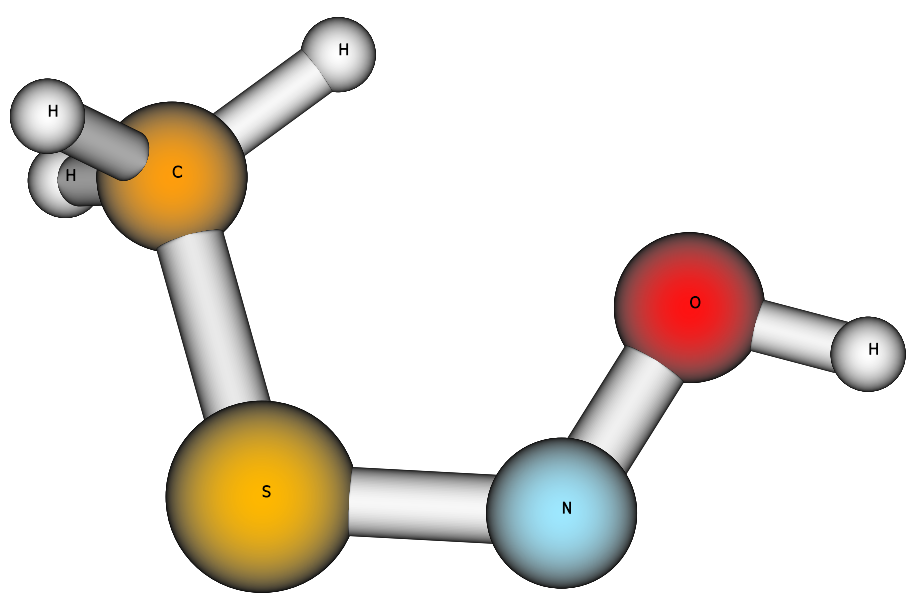

$\mathrm{CH}_{3}-\mathrm{SNO}\left(\mathrm{H}^{+}\right) \quad \mathrm{E}=-567.9522442$

$0,0,-1.1714232419,0 .,-1.0838498575$

$\mathrm{N}, 0,-1.1270978959,0 ., 0.2064452759$

$S, 0,0.325771112,0.0 .8591319248$

C, $0,1.4960015154,0 .,-0.4940572475$

$\mathrm{H}, 0,2.4879723717,0 .,-0.0280576344$

$\mathrm{H}, 0,1.3527272937,0.9030603615,-1.1010505313$

$\mathrm{H}, 0,1.3527272937,-0.9030603615,-1.1010505313$

$\mathrm{H}, \mathrm{O},-2.1207026359,0 .,-1.3259266868$ 


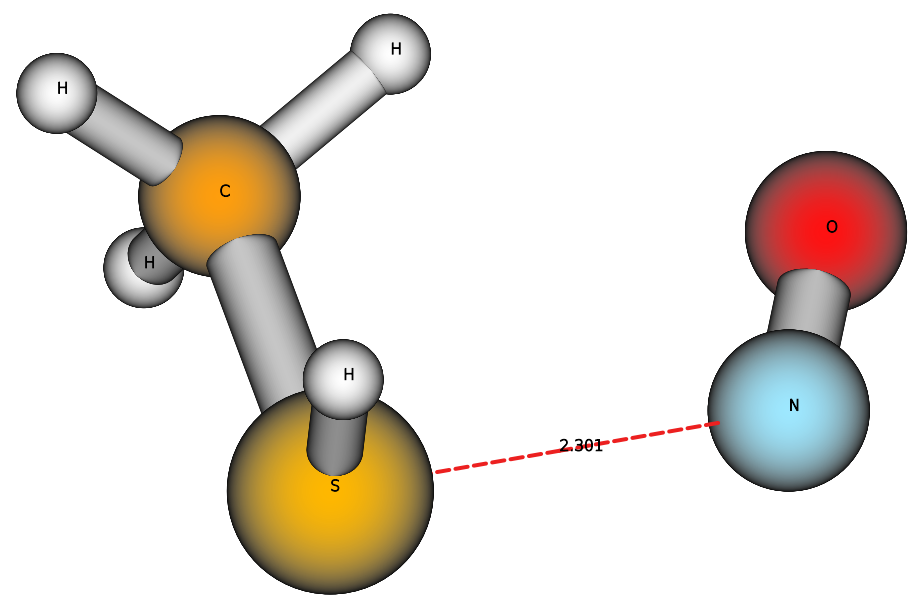

$\mathrm{CH}_{3}-\mathrm{S}\left(\mathrm{H}^{+}\right) \mathrm{NO} \quad \mathrm{E}=-567.9550351$

$0,1.7899514967,0.5521815815,0.0021456566$

$\mathrm{N}, 1.4655113437,-0.5068277883,0.0017185483$

$\mathrm{S},-0.8270969205,-0.6733038707,-0.09169206$

$\mathrm{C},-1.1453836826,1.0910350497,0.0178314064$

$\mathrm{H},-0.2364145197,1.6281825714,0.3337543843$

$\mathrm{H},-1.4496490772,1.425420259,-0.9783548023$

$\mathrm{H},-1.946561446,1.2774337782,0.7406119292$

$\mathrm{H},-0.8397135128,-0.9740431104,1.2348779199$ 


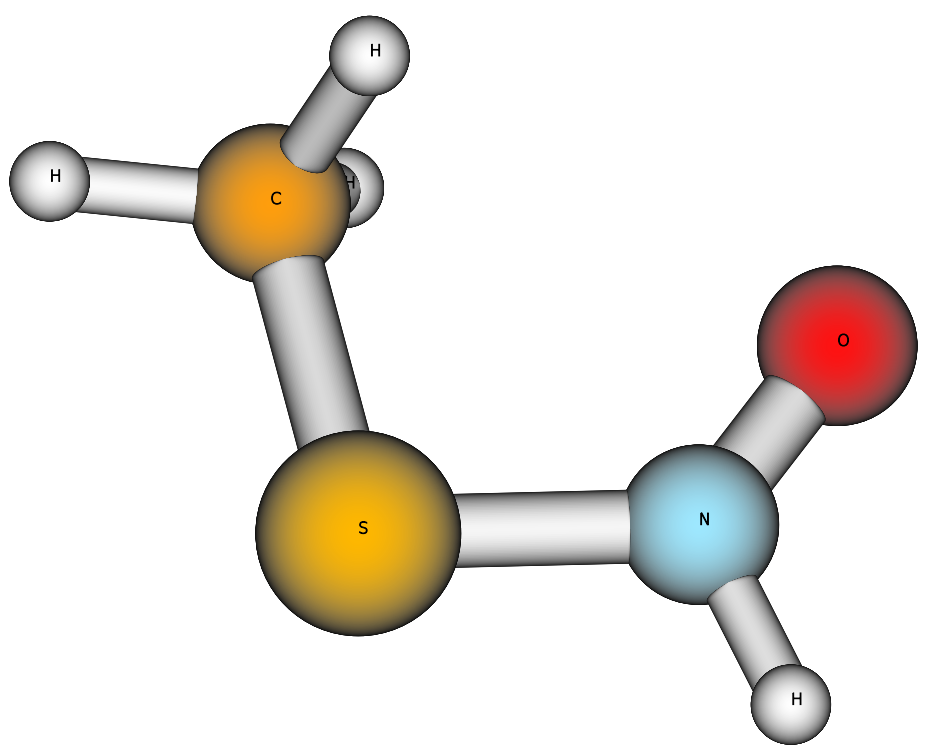

$\mathrm{CH}_{3}-\mathrm{SN}\left(\mathrm{H}^{+}\right) \bigcirc \mathrm{E}=-567.9535389$

$C, 0,-0.7747299962,0.0000000759,-1.3565322006$

$S, 0,-0.8328373048,-0.0000000453,0.4269985088$

$\mathrm{N}, 0,0.8169362928,-0.0000000336,0.7399835866$

$0,0,1.6938819598,0.0000000382,-0.0668212085$

$\mathrm{H}, 0,-1.8253397084,0.0000000763,-1.6717284266$

$\mathrm{H}, 0,-0.2674275086,-0.9063605382,-1.7095908975$

$\mathrm{H}, 0,-0.2674275446,0.9063607579,-1.7095907754$

$\mathrm{H}, 0,1.0643618876,-0.0000000969,1.7528117242$ 


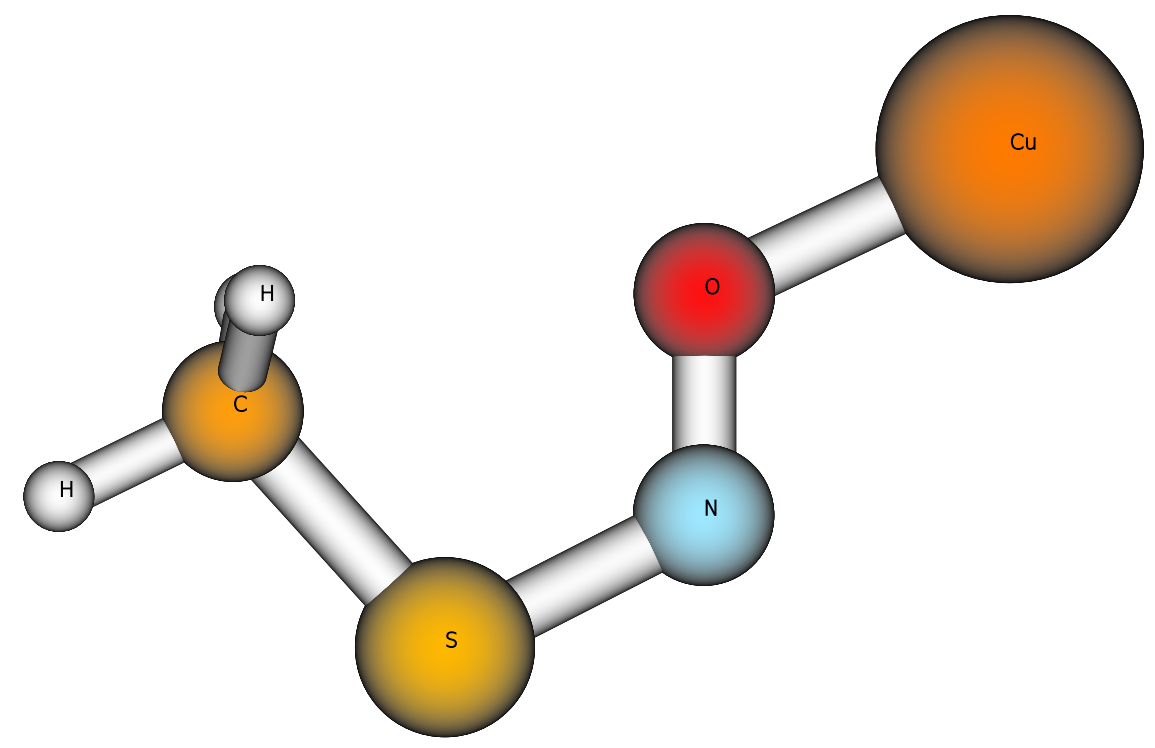

$\mathrm{CH}_{3}-\mathrm{SNO}\left(\mathrm{Cu}^{\mathrm{I}}\right) \quad \mathrm{E}=-764.6560915$

C, $0,-2.7110112092,-0.1431737633,0$.

$\mathrm{S}, 0,-1.6135595877,-1.5594472077,0$.

$\mathrm{N}, 0,-0.0982419015,-0.9243147464,0$.

$0,0,-0.0019439261,0.3232503546,0$.

$\mathrm{H}, 0,-3.7281176548,-0.5492171188,0$.

$\mathrm{H}, 0,-2.5415784055,0.4573243905,0.9007078273$

$\mathrm{H}, 0,-2.5415784055,0.4573243905,-0.9007078273$

$\mathrm{Cu}, 0,1.7792255118,1.0113436769,0$. 


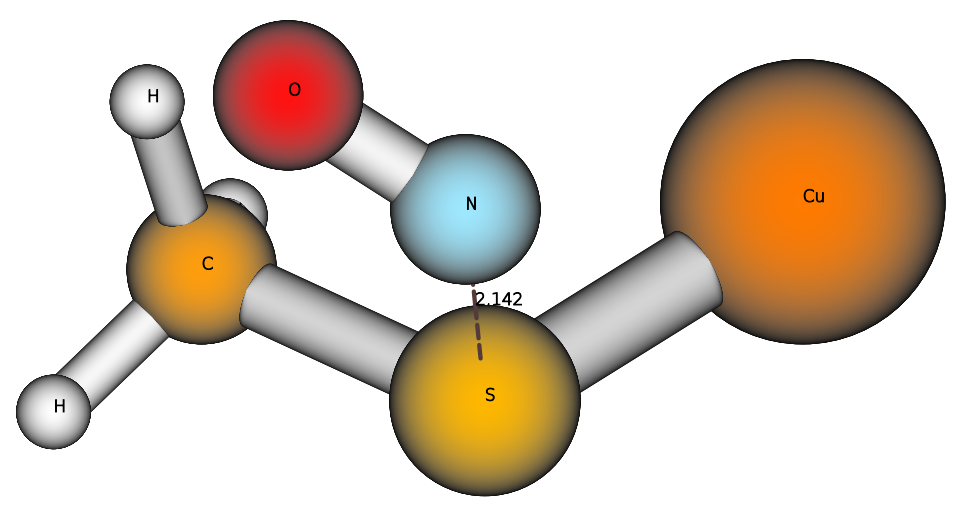

$\mathrm{CH}_{3}-\mathrm{S}\left(\mathrm{Cu}^{\mathrm{I}}\right) \mathrm{NO} \mathrm{E}=-764.6698295$

$S, 0,0.2959178663,0.4907059397,0.7058625923$

$\mathrm{N}, 0,1.5596381707,-1.1832703075,0.2728941714$

$\mathrm{C}, 0,1.0425655936,1.6366028444,-0.4683571182$

$\mathrm{H}, 0,0.2769060924,2.1971487678,-1.0105690971$

$\mathrm{H}, 0,1.653414876,1.0675251733,-1.1869638563$

$\mathrm{H}, 0,1.6833308419,2.3232191571,0.0934557031$

$0,0,2.3754168514,-0.9288357442,-0.4593303982$

$\mathrm{Cu}, 0,-1.5353287324,-0.2601802446,-0.1591447424$ 


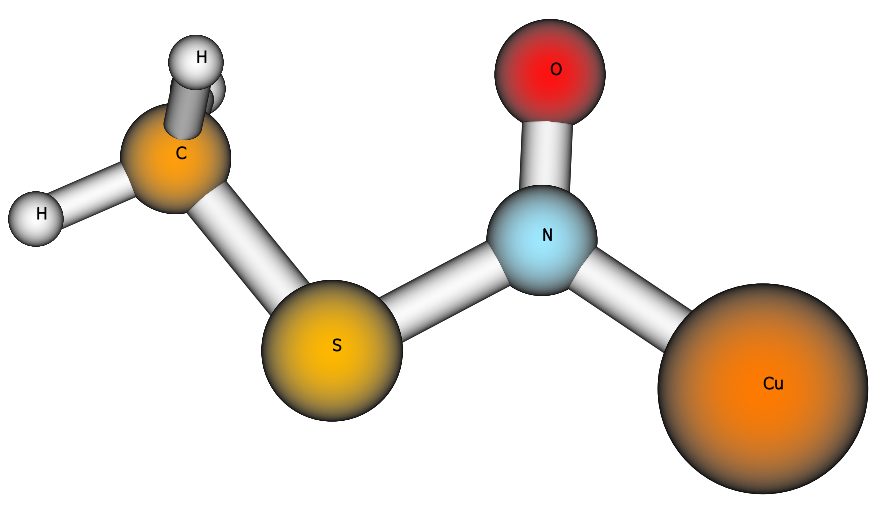

$\mathrm{CH}_{3}-\mathrm{SN}\left(\mathrm{Cu}^{\mathrm{I}}\right) \bigcirc \mathrm{E}=-764.6619027$

$\mathrm{S}, 1.4658659845,-0.4416411872,0$.

$\mathrm{N},-0.0142562933,0.4111752558,0$.

C, 2.6411502231,0.9056715255,0.

$\mathrm{H}, 3.6298377922,0.4326300149,0$.

$\mathrm{H}, 2.509714851,1.513056309,0.9022932927$

$\mathrm{H}, 2.509714851,1.513056309,-0.9022932927$

$0,-0.0280469018,1.6086483886,0$.

$\mathrm{Cu},-1.642270873,-0.6059974721,0$. 


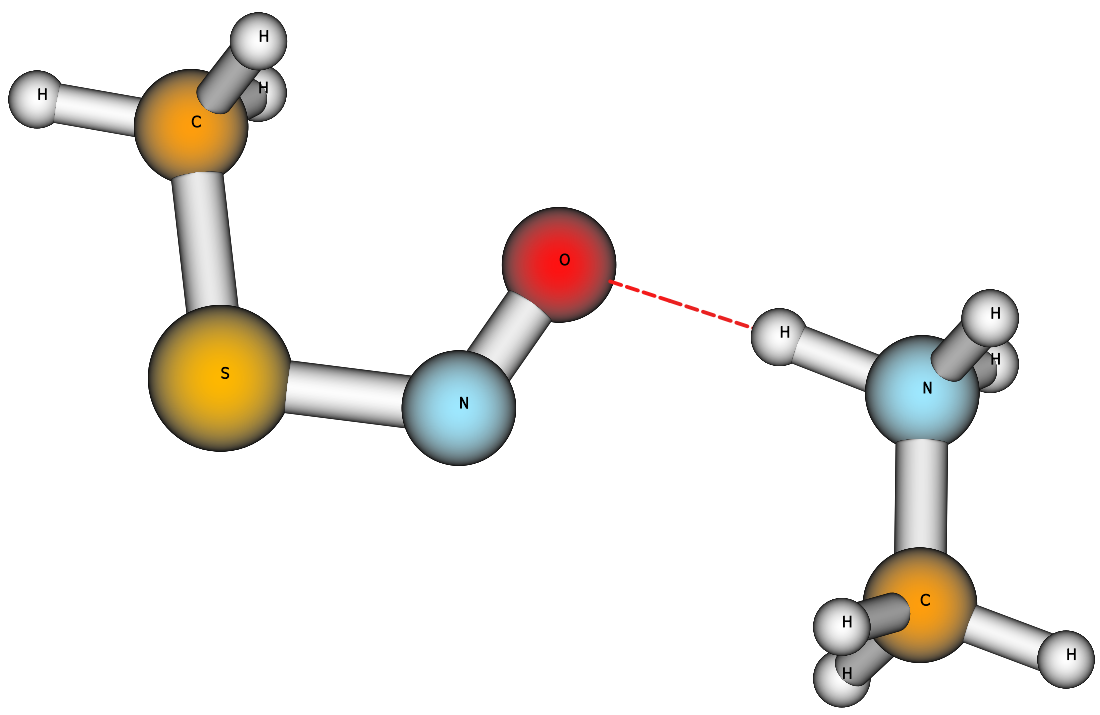

$\mathrm{CH}_{3}-\mathrm{SNO}\left(\mathrm{CH}_{3} \mathrm{NH}_{3}{ }^{+}\right) \quad \mathrm{E}=-663.7663459$

$\mathrm{C}, 0,2.6448362693,0.8122016106,-0.3988968424$

$\mathrm{S}, 0,1.3118309331,1.5069806702,0.5768067343$

$\mathrm{N}, 0,0.0780881126,0.383959538,0.4123941501$

$0,0,0.2965419238,-0.6087781356,-0.2781566458$

$\mathrm{N}, 0,-2.0350517332,-1.9431287789,-0.097135265$

$\mathrm{H}, 0,-1.0886364895,-1.460462079,-0.1910598278$

$\mathrm{H}, \mathrm{0},-2.1451600812,-2.2234490855,0.8797133154$

$\mathrm{H}, \mathrm{0},-2.0083313053,-2.8009454512,-0.6520975474$

$\mathrm{H}, 0,2.9339430385,-0.1629925415,0.007109676$

$\mathrm{H}, 0,3.4803261841,1.5156391858,-0.3246400009$

$\mathrm{H}, 0,2.3277333793,0.7097467473,-1.4421251973$

C, $0,-3.1399631309,-1.047673568,-0.5311146656$

$\mathrm{H}, 0,-4.0953002081,-1.5667344331,-0.4288395328$

$\mathrm{H}, 0,-3.1248235762,-0.153710006,0.0957685936$

$\mathrm{H}, 0,-2.9718747469,-0.7715415448,-1.5742272089$ 


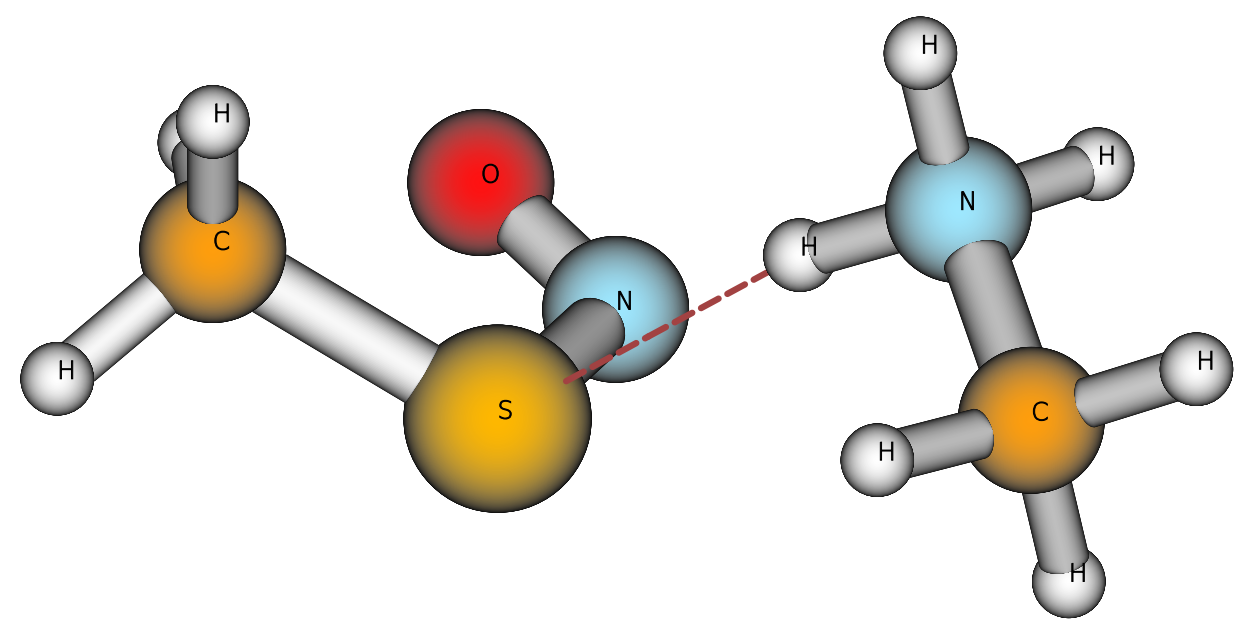

$\mathrm{CH}_{3}-\mathrm{S}\left(\mathrm{CH}_{3} \mathrm{NH}_{3}^{+}\right) \mathrm{NO} \mathrm{E}=-663.7550544$

C, $0,1.8463093707,1.1615306386,0.8942670751$

$S, 0,0.5260649863,0.8320851446,-0.271358603$

$\mathrm{N}, 0,1.0708989692,-0.906959676,-0.9381679934$

$0,0,2.0853834883,-1.2863223587,-0.5415909918$

$\mathrm{N}, 0,-2.2796563954,-0.1871256998,0.9154322008$

$\mathrm{H}, 0,-2.198394407,-0.950636631,1.5912824136$

$\mathrm{H}, 0,-2.7363609038,0.5931186866,1.3941213148$

$\mathrm{H}, 0,-1.3017336378,0.1237699493,0.6520891898$

$\mathrm{H}, 0,2.5376580826,0.3039475168,0.8501491059$

$\mathrm{H}, \mathrm{0}, 1.4610140595,1.2661408206,1.9124383862$

$\mathrm{H}, 0,2.3811767373,2.0670201936,0.5943183164$

$C, 0,-3.0374956402,-0.6050110144,-0.2956424607$

$\mathrm{H}, 0,-4.0411563437,-0.92088829,-0.0038983867$

$\mathrm{H}, 0,-3.0910609968,0.2453595936,-0.9788548968$

$\mathrm{H}, 0,-2.5028306764,-1.4311353989,-0.7697769988$ 


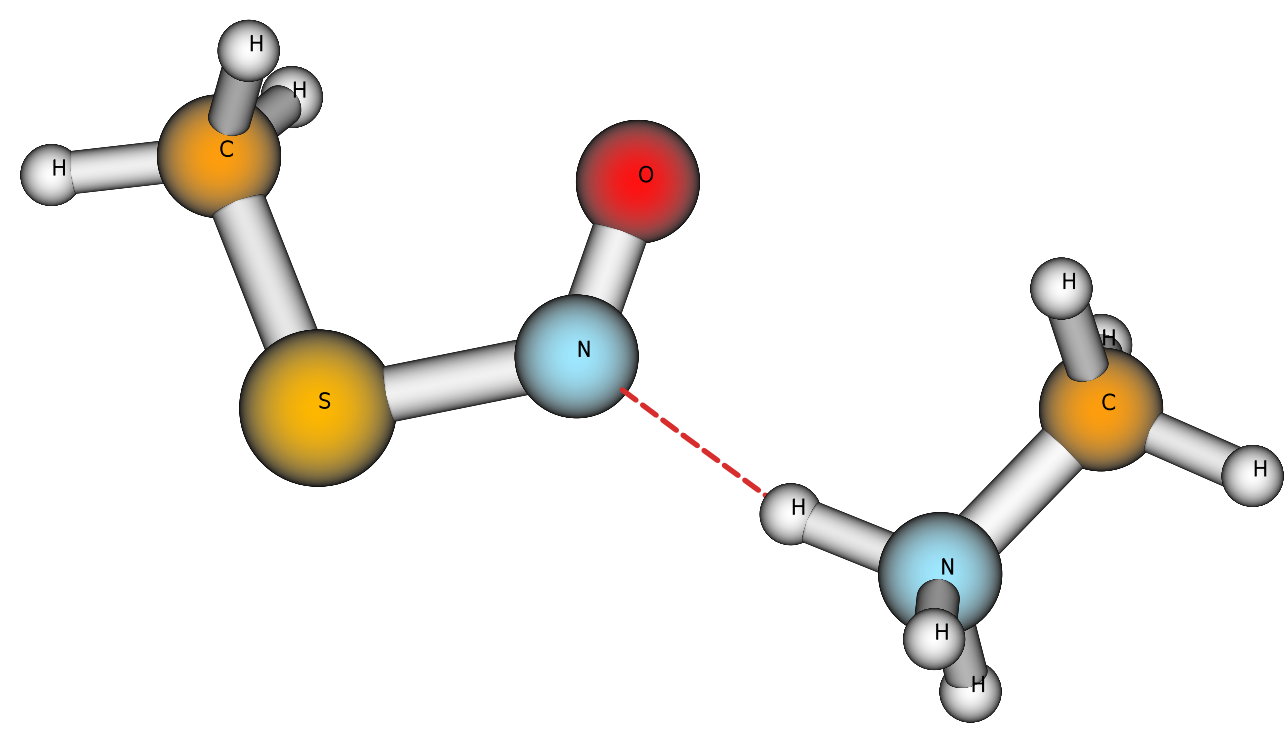

$\mathrm{CH}_{3}-\mathrm{SN}\left(\mathrm{CH}_{3} \mathrm{NH}_{3}{ }^{+}\right) \bigcirc \mathrm{E}=-663.7635464$

C, $1.3041544684,2.528711806,0.1533378658$

S, $0.0081968872,1.6933360371,-0.7578208031$

$\mathrm{N},-0.1053730157,0.2120250026,0.0851441271$

$0,0.6191616074,0.0150267064,1.0295742285$

$\mathrm{N},-1.3314377805,-2.2626576671,0.3367434056$

$\mathrm{H},-1.3370678402,-2.286838199,1.359357619$

$\mathrm{H},-1.042408041,-1.2841316413,0.0587136336$

$\mathrm{H},-2.2904570012,-2.4219015617,0.0212193397$

$\mathrm{H}, 0.9966445588,2.6661292884,1.1955258894$

$\mathrm{H}, 1.4428854901,3.4999714362,-0.3325487211$

$\mathrm{H}, 2.2293144936,1.9445150115,0.1046411125$

$\mathrm{C},-0.3916616478,-3.2717794557,-0.2212273441$

$\mathrm{H},-0.6996696158,-4.2703453088,0.0958351961$

$\mathrm{H},-0.4113590241,-3.2001100843,-1.3108150553$

$\mathrm{H}, 0.6103925753,-3.0480446363,0.150734149$ 


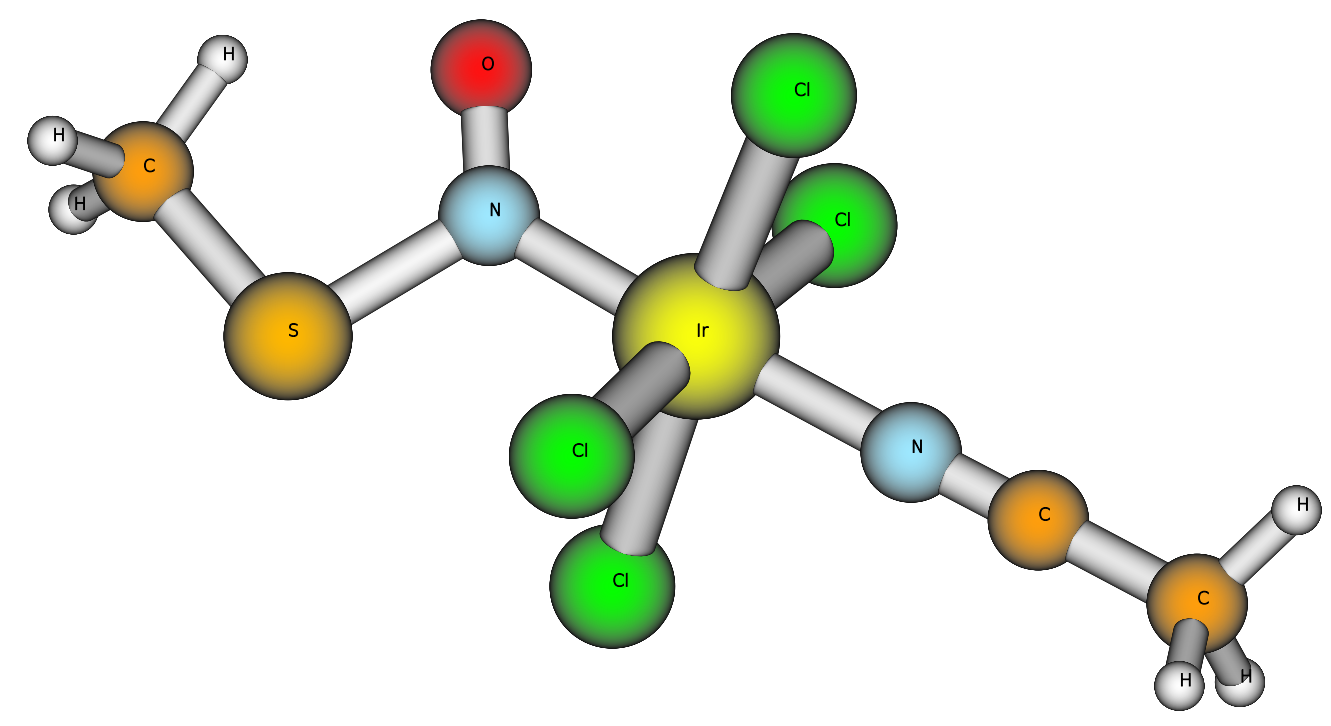

$\left[\mathrm{IrCl}_{4}\left(\mathrm{CH}_{3} \mathrm{CN}\right) \mathrm{N}(\mathrm{O}) \mathrm{SCH}_{3}\right]^{-} \mathrm{E}=-2645.3063467$

$\mathrm{H}, 0,3.259629929,-2.2250367241,-3.1809989888$

$\mathrm{C}, 0,2.3204339868,-1.6736262243,-3.3381373255$

$\mathrm{S}, 0,2.1158628067,-0.389679734,-2.0786238681$

$\mathrm{N}, 0,0.5445621754,-1.018999117,-1.316238408$

$0,0,0.050744654,-2.0327992701,-1.7702579005$

Ir $, 0,-0.1663680709,0.0258127597,0.1592042207$

$\mathrm{N}, 0,-0.941303959,1.0451837231,1.6667003729$

C, $0,-1.4051651881,1.6399187344,2.556097543$

$\mathrm{C}, 0,-1.9825748698,2.3818949384,3.6629174121$

$\mathrm{Cl}, 0,0.1865046659,2.1308262995,-0.988897014$

$\mathrm{Cl}, 0,2.0040029397,0.258958656,1.2125852781$

$\mathrm{Cl}, 0,-2.3854185476,-0.1288680568,-0.7946962643$

$\mathrm{Cl}, 0,-0.5631757272,-2.0062914042,1.4129389831$

$\mathrm{H}, 0,1.4633837468,-2.3631416776,-3.2167033582$

$\mathrm{H}, 0,2.29761096,-1.2346244476,-4.34689414$

$\mathrm{H}, \mathrm{0},-2.5530397317,3.2443288624,3.2820138531$

$\mathrm{H}, 0,-1.1868512082,2.7510250433,4.3299347544$

$\mathrm{H}, \mathrm{O},-2.6616520864,1.736086012,4.2426417404$ 


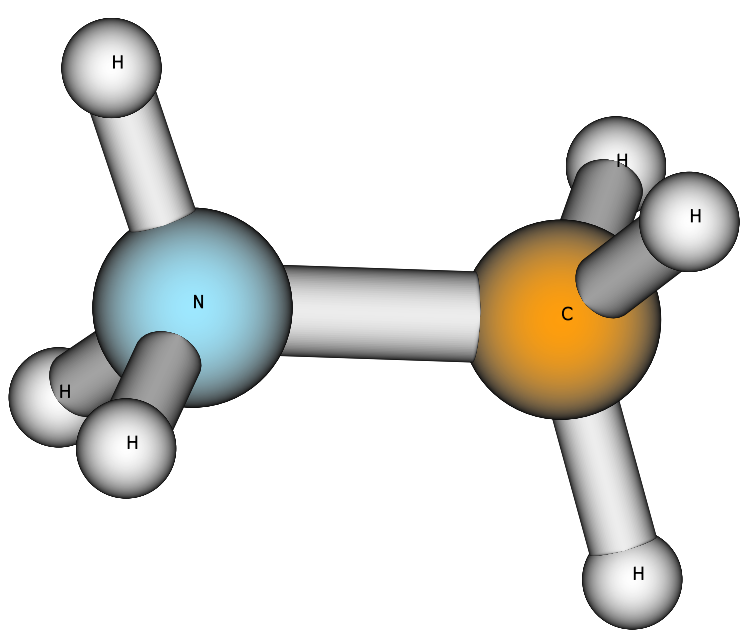

$\mathrm{CH}_{3} \mathrm{NH}_{3}{ }^{+} \mathrm{E}=-95.7291704$

$\mathrm{H}, 0,-0.7792526864,0 .,-1.289943469$

$\mathrm{C}, 0,-0.6756216575,0 .,-0.190934685$

$\mathrm{N}, 0,0.6922311165,0 ., 0.3043362951$

$\mathrm{H}, 0,1.1951011911,-0.8117481406,-0.0430208405$

$\mathrm{H}, 0,1.1951011911,0.8117481406,-0.0430208405$

$\mathrm{H}, 0,-1.2014187831,0.8813774078,0.1956195971$

$\mathrm{H}, 0,-1.2014187831,-0.8813774078,0.1956195971$

$\mathrm{Cu}^{+} \mathrm{E}=-196.9292369$ 